\title{
Impact of frailty markers on outcomes after transcatheter aortic valve replacement: insights from a Japanese multicenter registry
}

Tetsuro Shimura ${ }^{1}$, Masanori Yamamoto ${ }^{1,2}$, Seiji Kano ${ }^{2}$, Ai Kagase ${ }^{1}$, Atsuko Kodama ${ }^{1}$, Yutaka Koyama ${ }^{2}$, Toshiaki Otsuka ${ }^{3,4}$, Shun Kohsaka ${ }^{5}$, Norio Tada $^{6}$, Futoshi Yamanaka ${ }^{7}$, Toru Naganuma ${ }^{8}$, Motoharu Araki $^{9}$, Shinichi Shirai ${ }^{10}$, Kazuki Mizutani ${ }^{11}$, Minoru Tabata ${ }^{12}$, Hiroshi Ueno ${ }^{13}$, Kensuke Takagi ${ }^{14}$, Akihiro Higashimori $^{15}$, Yusuke Watanabe ${ }^{16}$, Kentaro Hayashida ${ }^{5}$; on the behalf of OCEAN-TAVR investigators

${ }^{1}$ Department of Cardiology, Toyohashi Heart Center, Toyohashi, Japan; ${ }^{2}$ Department of Cardiology, Nagoya Heart Center, Nagoya, Japan; ${ }^{3}$ Department of Hygiene and Public Health, Nippon Medical School, Tokyo, Japan; ${ }^{4}$ Center for Clinical Research, Nippon Medical School Hospital, Tokyo, Japan; ${ }^{5}$ Department of Cardiology, Keio University School of Medicine, Tokyo, Japan; ${ }^{6}$ Department of Cardiology, Sendai Kousei Hospital, Sendai, Japan; ${ }^{7}$ Department of Cardiology, Shonan Kamakura General Hospital, Kamakura, Japan; ${ }^{8}$ Department of Cardiology, New Tokyo Hospital, Chiba, Japan; ${ }^{9}$ Department of Cardiology, Saiseikai Yokohama City Eastern Hospital, Yokohama, Japan; ${ }^{10}$ Department of Cardiology, Kokura Memorial Hospital, Kokura, Japan; ${ }^{11}$ Department of Cardiology, Osaka City University Hospital, Osaka, Japan; ${ }^{12}$ Department of Cardiology, Tokyo Bay Urayasu Ichikawa Medical Center, Urayasu, Japan; ${ }^{13}$ Department of Cardiology, Toyama University Hospital, Toyama, Japan; ${ }^{14}$ Department of Cardiology, Ogaki Municipal Hospital, Ogaki, Japan; ${ }^{15}$ Department of Cardiology, Kishiwada Tokushukai Hospital, Kishiwada, Japan; ${ }^{16}$ Department of Cardiology, Teikyo University School of Medicine, Tokyo, Japan

Correspondence to: Masanori Yamamoto, MD, PhD, FESC. Department of Cardiology, Toyohashi Heart Center, 21-1 Gobutori, Oyama-Cho, Toyohashi, Aichi 441-8530, Japan. Email: masa-nori@nms.ac.jp; yamamoto@heart-center.or.jp.

There are no standardized criteria for measuring patients' frailty. We examined prognosis based on four frailty markers [serum albumin level, grip strength, gait speed, and clinical frailty scale (CFS)] in patients who underwent transcatheter aortic valve replacement (TAVR) between October 2013 and April 2016 and were recorded in the Optimized CathEter vAlvular iNtervention (OCEAN) Japanese multicenter registry. Serum albumin level was assessed by dividing patients into two groups: hypoalbuminemia or non-hypoalbuminemia according to their serum albumin level. Clinical outcomes including all-cause, cardiovascular and noncardiovascular mortality rates after TAVR were compared. During the follow-up period cumulative all-cause, cardiovascular and non-cardiovascular mortality rates were significantly higher in the hypoalbuminemia group than in the non-hypoalbuminemia group. This result remained unchanged even after a propensitymatched model was used in terms of cumulative all-cause and non-cardiovascular mortality; however, differences in cardiovascular mortality rates were attenuated. To consider the impact of grip strength patients were divided into a low or high peak grip strength group based on classification and regression tree (CART) survival analysis. The clinical outcomes for each sex were compared between the two groups. In both sexes the cumulative 1-year mortality rates were significantly different between the two groups. To investigate gait speed patients were classified into two gait speed groups (low or high gait speed group) based on CART survival analysis. Clinical outcomes were compared between the two groups. The cumulative 1-year mortality rate was significantly different between the two gait speed groups. The effect of CFS on prognosis after TAVR was assessed. Patients were categorized into five groups based on the following CFS scores: CFS1-3, CFS4, CFS5, CFS6, and CFS $\geq 7$. We evaluated the relationship between the CFS score and other indicators of frailty markers. We also assessed the mid-term mortality among the five groups. The CFS score had a significant correlation with other frailty markers. The cumulative 1-year mortality increased with an increasing CFS score. In the Cox regression multivariable analysis, the CFS score was an independent predictive factor of an increased late cumulative mortality risk. In conclusion, the results suggest that serum albumin level, grip strength, gait speed, and CFS score are all useful indicators when considering the optimal indications and risk stratification for TAVR. 
Keywords: Transcatheter aortic valve replacement (TAVR); albumin; grip strength; gait speed; clinical frailty scale (CFS); frailty; Optimized CathEter vAlvular iNtervention (OCEAN)

Submitted Jul 27, 2017. Accepted for publication Aug 30, 2017.

doi: $10.21037 /$ acs.2017.09.06

View this article at: http://dx.doi.org/10.21037/acs.2017.09.06

\section{Introduction}

The 2017 American Heart Association (AHA)/American College of Cardiology (ACC) guideline for valvular heart disease (VHD) gives a class I recommendation for the indication of transcatheter aortic valve replacement (TAVR) in patients with severe aortic stenosis (AS) for whom surgical aortic valve replacement (SAVR) was considered a high risk (1). It is thought that this recommendation may increase the number of patients who will undergo TAVR. However, most patients with severe AS who are considered high risk for SAVR are of advanced aged. When examining the prognosis of such a cohort, it is necessary to evaluate patient frailty in addition to surgical risk scores (2). In the 2014 AHA/ACC guideline for VHD, the Katz index and gait speed have been recommended as good assessment tools of patient frailty (3), although there are no standardized criteria for measuring patient frailty. Recently, several studies on a cohort of patients who underwent TAVR reported that patient frailty is strongly associated with long-term prognosis $(4,5)$. However, all these studies were performed in a small number of patients at a single center. Further investigations using a multicenter registry are required.

We [the Optimized CathEter vAlvular iNtervention (OCEAN)-SHD group] examined prognosis based on the serum albumin level, grip strength, gait speed, and clinical frailty scale (CFS) score in patients who underwent TAVR between October 2013 and April 2016 using the OCEANTAVI registry as a multicenter study of a high-volume center of TAVR in Japan.

\section{Serum albumin}

We evaluated the serum albumin level for predicting prognosis after TAVR. The recently updated Valve Academic Research Consortium (VARC)-2 highlighted the importance of frailty, including the serum albumin level (6). According to these criteria, a serum albumin level less than $3.5 \mathrm{~g} / \mathrm{dL}$ is a new risk factor reflecting frailty, which may be associated with a worse prognosis.
We divided 1,215 patients from nine centers undergoing TAVR into two groups according to their serum albumin levels: patients with hypoalbuminemia (hALB group, albumin level $<3.5 \mathrm{~g} / \mathrm{dL}$ ) or patients without hypoalbuminemia (nhALB group, albumin level $\geq 3.5 \mathrm{~g} / \mathrm{dL}$ ). Baseline characteristics and clinical outcomes, including all-cause, cardiovascular, and noncardiovascular mortality rates after TAVR, were compared between the two groups. There were differences between the hALB and nhALB groups in terms of age, body characteristics, the prevalence of New York Heart Association (NYHA) class III or IV, liver disease, estimated glomerular filtration rate, hemoglobin level, and left ventricular ejection fraction. An evaluation of preoperative risk factors showed significant differences in the logistic European System for Cardiac Operative Risk Evaluation (logistic EuroSCORE) and Society of Thoracic Surgeons Predictive Risk of Mortality risk (STS) score. Moreover, there were differences between the hALB and nhALB groups in terms of liver disease, estimated glomerular filtration rate, and the presence of a malignant tumor, which may affect the serum albumin level. Therefore, propensityscore matching was selected as a method to provide a valid comparison between the hALB and nhALB groups. After propensity-score matching, baseline patient characteristics in the two matching models were closely adjusted, except for the serum albumin level.

Kaplan-Meier curves showed significantly increased allcause, cardiovascular, and non-cardiovascular mortality rates in the hALB group compared to those in the nhALB group. The analysis after propensity-score matching revealed that the poorer prognosis of the hALB group in terms of cumulative all-cause and non-cardiovascular mortality remained. However, differences in cardiovascular mortality rates between the two groups were attenuated.

The results of this study demonstrated that the preprocedural serum albumin concentration could be a useful marker for risk stratification before TAVR (7).

\section{Grip strength}

We analyzed dominant-hand grip strength as a clinical 
prognostic indicator after TAVR. The dominant-hand grip strength is a simple marker that reflects frailty associated with incremental risks for late mortality (8-11).

Of 1,215 patients from nine centers, we initially excluded 241 patients with missing grip strength data. As a result, the remaining 927 patients were included in this study population. The classification and regression tree (CART) analysis (an empirical and statistical method used to create the decision rules based on data rather than speculation and to make the risk stratification model) identified the optimal thresholds of peak grip strength for each sex (12). We divided patients into two risk groups (the low and high peak grip groups) according to the aforementioned cutoff values. Thereafter, baseline characteristics and all-cause mortality, cardiovascular mortality, and non-cardiovascular mortality rates were compared between the two groups for each sex.

There were statistical differences in age, sex, body characteristics, the prevalence of NYHA class III or $\mathrm{IV}$, B-type natriuretic peptide value (BNP), and other comorbidities. The logistic-EuroSCORE, EuroSCORE II, and STS score were higher in the low peak grip groups, regardless of sex differences. The Kaplan-Meier curves showed significant differences in each group in terms of all-cause mortality. Among men, the cumulative 1-year mortality rate was significantly higher in the low peak grip group than in the high peak grip group. Among women, a higher cumulative 1 -year mortality rate was found in the low grip strength group than in the high grip strength group. Kaplan-Meier curves indicated no significant increased cardiovascular mortality rates between the two groups. However, non-cardiovascular mortality rates indicated significant differences between the two groups in both sexes.

The results of the current study demonstrated that peak grip strength was a useful marker for predicting all-cause mortality following TAVR, particularly with non-cardiac deaths (13).

\section{Gait speed}

Gait speed was evaluated. It has been previously proven that gait speed is important as a frailty marker relevant to cardiovascular surgery, including TAVR (2).

Our study enrolled 1,613 patients from 14 institutions. Initially, we excluded 262 patients with missing data. The final sample included the remaining 1,256 patients. Patients were divided two risk groups (low and high gait speed groups) of gait speed classification according to the cutoff values obtained from the CART analysis. Baseline characteristics, procedural outcomes, and all-cause, cardiovascular, and non-cardiovascular mortality rates were compared among the four groups.

Statistically significant differences were found between the groups for age, sex, the prevalence of NYHA class III or IV, and other comorbidities. As a result, preoperative risk scores such as the Logistic EuroSCORE, EuroSCORE II, and STS score were higher in patients with low gait speed. The procedure time was significantly different between two groups. The approach route was also significantly different between the two groups, with the rate for the nontransfemoral approach higher in low gait speed group. In addition, significant group differences were found for the length of hospital stay, VARC-2 defined acute kidney injury and life-threatening or disabling bleeding. The 30-day mortality rates were not statistically different among the groups.

The Kaplan-Meier curves demonstrated significant difference between two groups in terms of all-cause mortality and non-cardiovascular mortality. However, Kaplan-Meier curves indicated no significant increase in cardiovascular mortality rates between the two gait speed groups.

Our results demonstrated that gait speed is useful as a potential marker for predicting vulnerable patients associated with advanced clinical outcomes following TAVR (14).

\section{Clinical frailty score}

We assessed the clinical frailty score as a marker/substitute for general patient activity. The CFS is a simple tool for assessing patient's frailty and has been shown to be correlated with mortality in elderly patients even when evaluated by non-geriatricians $(15,16)$.

We analyzed data for 1,215 consecutive patients with symptomatic severe AS who underwent TAVR and were available from the OCEAN-TAVI registry. All CFS scores were calculated during face-to-face assessments with patients and families to determine the baseline frailty status before TAVR. The CFS score ranged from 1 (very fit) to 9 (terminally ill). Detailed CFS criteria are presented in Table 1. The CFS scores were categorized into five groups as follows: non-frail (CFS 1-3), vulnerable (CFS 4), mildly frail (CFS 5), moderately frail (CFS 6), and severely frail $(\mathrm{CFS} \geq 7)$. We assessed the differences between in baseline characteristics, clinical outcomes including early and 


\begin{tabular}{|c|c|c|}
\hline 1 & Very fit & $\begin{array}{l}\text { People who are robust, active, energetic and motivated. These people commonly exercise regularly. They are } \\
\text { among the fittest for their age }\end{array}$ \\
\hline 2 & Well & $\begin{array}{l}\text { People who have no active disease symptoms but are less fit than category } 1 \text {. Often, they exercise or are } \\
\text { very active occasionally, e.g., seasonally }\end{array}$ \\
\hline 4 & Vulnerable & $\begin{array}{l}\text { While not dependent on others for daily help, often symptoms limit activities. A common complaint is being } \\
\text { "sloped up", and/or being tired during the day }\end{array}$ \\
\hline 5 & Mildly frail & $\begin{array}{l}\text { These people often have more evident slowing, and need help in high older IADLs (finances, transportation, } \\
\text { heavy housework, medications). Typically, mild frailty progressively impairs shopping and walking outside } \\
\text { alone, meal preparation and housework }\end{array}$ \\
\hline 7 & Severely frail & $\begin{array}{l}\text { Completely dependent for personal care, from whatever cause (physical or cognitive). Even so, they seem } \\
\text { stable and not at high risk of dying (within } ~ 6 \text { months) }\end{array}$ \\
\hline 8 & Very severely frail & Completely dependent, approaching the end of life. Typically, they could not recover from a minor illness \\
\hline 9 & Terminally ill & $\begin{array}{l}\text { Approaching the end of life. This category applies to people with a life expectancy }<6 \text { months, who are not } \\
\text { otherwise evidently frail }\end{array}$ \\
\hline
\end{tabular}

midterm mortality among the five groups. Additional frailty factors including albumin level, body mass index (BMI), gait speed ( $\mathrm{m} / \mathrm{sec}$ - calculated on a 15 -foot or 5 -m walk) and mean grip strength $(\mathrm{kg})$ were evaluated. Univariate Cox regression analysis was performed to obtain the hazard ratio for late mortality during the follow-up period. Multivariable analysis was performed using the baseline clinical characteristics and other variables to examine their independent associations with late mortality.

Among the five study groups, significant differences were observed in the mean age, sex, height, weight, BMI, Logistic EuroSCORE, and STS score. A significant finding was that serum albumin and hemoglobin levels were lowest in the severely frail group. The associations between each of the four components of frailty and CFS score differed significantly among the five groups. Moreover, we identified significant correlations between the CFS score and BMI, albumin level, gait speed, and mean grip strength. The 30-day mortality and in-hospital mortality rates were not significantly different among the groups. However, the CFS score increment was significantly associated with an increasing risk of 30-day mortality. Kaplan-Meier analysis of the cumulative 1-year mortality rates slightly increased across the CFS1-3, CFS 4, CFS 5, and CFS 6 groups, and a significant increase was observed in patients with CFS $\geq 7$ compared with all other groups. Cox regression analysis models examining the association between late mortality, CFS score and other frailty markers demonstrated such markers were significantly associated with an increasing risk of late cumulative mortality. These results were not attenuated- even after adjustment for multiple confounding factors in the Cox regression multivariable analysis.

Although the CFS grading tool is disadvantageous by its semi-quantitative classification, our study identified a positive correlation between the CFS score and several other indicators of frailty defined by the VARC-2 criteria (6). As a result, the CFS is a useful predictor of short-term and mid-term mortality in patients who undergo TAVR (17).

\section{Conclusions}

On the basis of the results of our study, we reconfirmed the importance and clinical use of frailty markers in a large number of patients from a multicenter registry. The summary of frailty tools and 1-year cumulative mortality, which we discussed in this paper, is presented in Figure 1. Aside from the four aforementioned frailty items, there are several other frailty markers, such as anemia and BMI 


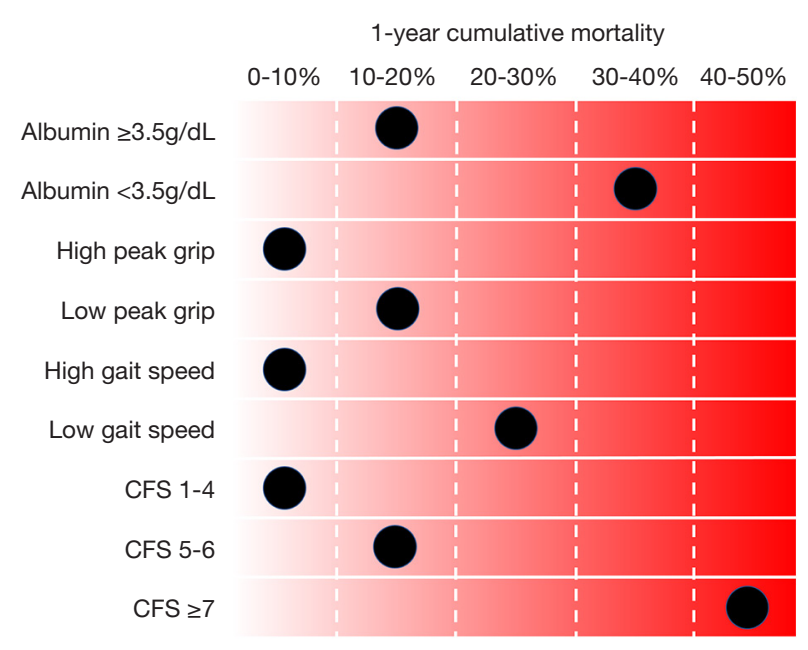

Figure 1 One-year cumulative mortality.

$<20 \mathrm{~kg} / \mathrm{m}^{2}$, that are considered useful. It is also recognized that a combination of specific frailty markers enables physicians to formulate a more accurate prognosis. However, it is difficult to decide which frailty item should be used. In the future, it would be beneficial if a frailty risk model was created using these markers. Until the creation of such a model, the CFS score seems to be one of the best frailty markers for predicting patients' prognosis after TAVR. Categorization by CFS score is simple to do for any condition, and the CFS score significantly correlates with other frailty markers. To identify suitable TAVR candidates, frailty markers, as aforementioned, will positively affect their clinical care and improve post-procedural outcomes.

\section{Acknowledgements}

None.

\section{Footnote}

Conflicts of Interest: The authors have no conflicts of interest to declare.

\section{References}

1. Nishimura RA, Otto CM, Bonow RO, et al. 2017 AHA/ ACC Focused Update of the 2014 AHA/ACC Guideline for the Management of Patients With Valvular Heart Disease: A Report of the American College of Cardiology/ American Heart Association Task Force on Clinical Practice Guidelines. J Am Coll Cardiol 2017;70:252-89.
2. Afilalo J, Eisenberg MJ, Morin JF, et al. Gait speed as an incremental predictor of mortality and major morbidity in elderly patients undergoing cardiac surgery. J Am Coll Cardiol 2010;56:1668-76.

3. Nishimura RA, Otto CM, Bonow RO, et al. 2014 AHA/ ACC Guideline for the Management of Patients With Valvular Heart Disease: a report of the American College of Cardiology/American Heart Association Task Force on Practice Guidelines. Circulation 2014;129:e521-643.

4. Green P, Woglom AE, Genereux P, et al. The impact of frailty status on survival after transcatheter aortic valve replacement in older adults with severe aortic stenosis: a single-center experience. JACC Cardiovasc Interv 2012;5:974-81.

5. Puls M, Sobisiak B, Bleckmann A, et al. Impact of frailty on short- and long-term morbidity and mortality after transcatheter aortic valve implantation: risk assessment by Katz Index of activities of daily living. EuroIntervention 2014;10:609-19.

6. Kappetein AP, Head SJ, Généreux P, et al. Updated standardized endpoint definitions for transcatheter aortic valve implantation: the Valve Academic Research Consortium-2 consensus document. Eur Heart J 2012;33:2403-18.

7. Yamamoto M, Shimura T, Kano S, et al. Prognostic Value of Hypoalbuminemia After Transcatheter Aortic Valve Implantation (from the Japanese Multicenter OCEANTAVI Registry). Am J Cardiol 2017;119:770-7.

8. Lawman HG, Troiano RP, Perna FM, et al. Associations of Relative Handgrip Strength and Cardiovascular Disease Biomarkers in U.S. Adults, 2011-2012. Am J Prev Med 2016;50:677-83.

9. Chen PJ, Lin MH, Peng LN, et al. Predicting causespecific mortality of older men living in the Veterans home by handgrip strength and walking speed: a 3-year, prospective cohort study in Taiwan. J Am Med Dir Assoc 2012;13:517-21.

10. Sasaki H, Kasagi F, Yamada M, et al. Grip strength predicts cause-specific mortality in middle-aged and elderly persons. Am J Med 2007;120:337-42.

11. Rantanen T, Volpato S, Ferrucci L, et al. Handgrip strength and cause-specific and total mortality in older disabled women: exploring the mechanism. J Am Geriatr Soc 2003;51:636-41.

12. Fonarow GC, Adams KF Jr, Abraham WT, et al. Risk stratification for in-hospital mortality in acutely decompensated heart failure: classification and regression tree analysis. JAMA 2005;293:572-80. 
13. Kagase A, Yamamoto M, Shimura T, et al. Gender-specific grip strength after transcatheter aortic valve replacement in elderly patients. JACC Cardiovasc Interv 2017. In Press.

14. Kano S, Yamamoto M, Shimura T, et al. Gait speed can predict advanced clinical outcomes in patients who undergo transcatheter aortic valve replacement: Insights from a Japanese multicenter registry. Circ Cardiovasc Interv 2017. In Press. doi: 10.1161/ CIRCINTERVENTIONS.117.005088.

15. Clinical Frailty Scale - Geriatric Medicine Research Dalhousie University. Faculty of Medicine, Geriatric

Cite this article as: Shimura T, Yamamoto M, Kano S, Kagase A, Kodama A, Koyama Y, Otsuka T, Kohsaka S, Tada N, Yamanaka F, Naganuma T, Araki M, Shirai S, Mizutani K, Tabata M, Ueno H, Takagi K, Higashimori A, Watanabe Y, Hayashida K; on the behalf of OCEAN-TAVR investigators. Impact of frailty markers on outcomes after transcatheter aortic valve replacement: insights from a Japanese multicenter registry. Ann Cardiothorac Surg 2017;6(5):532-537. doi: 10.21037/ acs.2017.09.06
Medicine Research, Research / Projects, Clinical Frailty Scale @. Dalhousie University, Halifax, Canada. Page last modified: 07/15/2015 22:47:07. Available online: http:// geriatricresearch.medicine.dal.ca/clinical_frailty_scale. htm. Accessed 2017-07-19.

16. Rockwood K, Song X, MacKnight C, et al. A global clinical measure of fitness and frailty in elderly people. CMAJ 2005;173:489-95.

17. Shimura T, Yamamoto M, Kano S, et al. Impact of the Clinical Frailty Scale on Outcomes After Transcatheter Aortic Valve Replacement. Circulation 2017;135:2013-24. 